\title{
Magnetic Design and Code Benchmarking of the SMC (Short Model Coil) Dipole Magnet
}

Pierre Manil, Federico Regis, James Rochford, Paolo Fessia, Simon Canfer, Elwyn Baynham, François Nunio, Gijs de Rijk, and Pierre Védrine

\begin{abstract}
The Short Model Coil (SMC) working group was set in February 2007 within the Next European Dipole (NED) program, in order to develop a short-scale model of a $\mathrm{Nb}_{3} \mathrm{Sn}$ dipole magnet. The SMC group comprises four laboratories: CERN/TE-MSC group (CH), CEA/IRFU (FR), RAL (UK) and LBNL (US). The SMC magnet is designed to reach a peak field of about 13 Tesla $(T)$ on conductor, using a $2500 \mathrm{~A} / \mathrm{mm}^{2}$ Powder-In-Tube (PIT) strand. The aim of this magnet device is to study the degradation of the magnetic properties of the $\mathrm{Nb}_{3} \mathrm{Sn}$ cable, by applying different levels of pre-stress. To fully satisfy this purpose, a versatile and easy-to-assemble structure has been realized. The design of the SMC magnet has been developed from an existing dipole magnet, the SD01, designed, built and tested at LBNL with support from CEA. The goal of the magnetic design presented in this paper is to match the high field region with the high stress region, located along the dipole straight section. For this purpose, three-dimensional nonlinear parametric models have been implemented using three codes (CAST3M, ANSYS, and OPERA). This optimization process has been an opportunity to cross-check the codes. The results of this benchmarking are presented here, along with the final design which incorporates the use of end spacers and a surrounding iron structure to deliver a nominal field of $13 \mathrm{~T}$ uniformly distributed along the cable straight section.
\end{abstract}

Index Terms-Dipoles, magnetic design, models cross-check, $\mathrm{Nb}_{3} \mathrm{Sn}$, superconducting accelerator magnets.

\section{INTRODUCTION}

$\mathbf{F}$ UTURE dipole magnet upgrades, requiring higher fields in larger apertures, will demand the use of a superconductor with greater performance compared to $\mathrm{Nb}-\mathrm{Ti}$, such as $\mathrm{Nb}_{3} \mathrm{Sn}$. This material is brittle and strain-sensitive. Its upper stress limit is estimated to be approximately $150 \mathrm{MPa}$, but the behavior of $\mathrm{Nb}_{3} \mathrm{Sn}$ insulated cables in coils is not fully understood. Improving this knowledge is essential for magnet designers because the higher magnetic fields generate large Lorentz forces and large stresses on the coil. The goal of the $\mathrm{SMC}$ working group is to create a subscale $\mathrm{Nb}_{3} \mathrm{Sn}$ dipole test bed where different levels of pre-stress can be applied to the

Manuscript received October 16, 2009. First published March 01, 2010; current version published May 28, 2010.

P. Manil and F. Nunio are with CEA Saclay/IRFU/SIS, 91191 Gif-sur-Yvette, France (e-mail: pierre.manil@cea.fr).

P. Védrine is with CEA Saclay/IRFU/SACM, 91191 Gif-sur-Yvette, France.

F. Regis, P. Fessia, and G. de Rijk are with CERN (European Organization for Nuclear Research), Switzerland (e-mail: federico.regis@cern.ch).

J. Rochford, S. Canfer, and E. Baynham are with RAL/STFC, Harwell Science and Innovation Campus, Didcot, U.K. (e-mail: james.rochford@stfc.ac. uk).

Color versions of one or more of the figures in this paper are available online at http://ieeexplore.ieee.org.

Digital Object Identifier 10.1109/TASC.2009.2039343

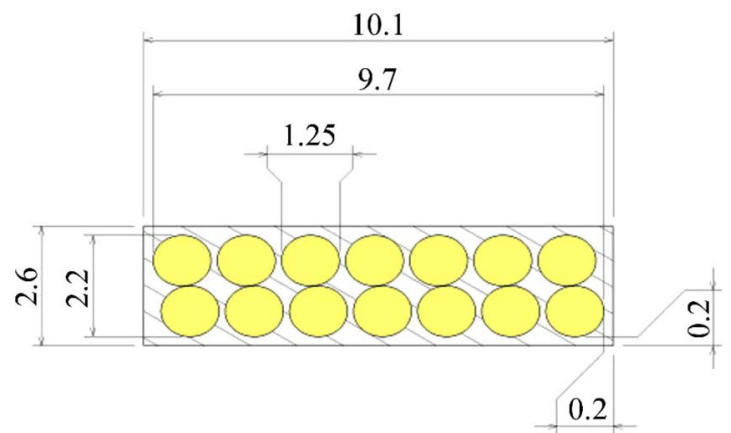

Fig. 1. Schematic view of the SMC cable cross-section (values in $\mathrm{mm}$ ).

coil. Varying the pre-stress makes it possible to explore both the mechanical limit of the $\mathrm{Nb}_{3} \mathrm{Sn}$ before degradation and the impact of a low pre-stress on the magnet training performance.

Several $\mathrm{Nb}_{3} \mathrm{Sn}$ magnets have been tested in the US since 1997 [1]-[4] within the High Field Magnets and LARP programs. In Europe, in January 2008, the HFM (High Field Magnets) took over the NED program, which was mainly focused on the $\mathrm{Nb}_{3} \mathrm{Sn}$ cable development [5]-[7]. Within the framework of the European project EuCARD, the SMC is the first European test dipole using the $\mathrm{Nb}_{3} \mathrm{Sn}$ conductor. It takes over the same design principles as the SD01 [8], a racetrack dipole coil tested at LBNL.

As for SD01, the SMC subscale dipole is racetrack-shaped. It is made of two $\mathrm{Nb}_{3} \mathrm{Sn}$ Rutherford cable layers wound around a metallic pole (double pancake configuration). A thin insulation sheet is placed between both pancakes. The whole dipole magnet consists in two double pancakes separated by a midplane insulation layer made of glass-fiber epoxy composite. The racetrack configuration makes the winding process and the prestress application simpler. Before magnetization, the coil pack is inserted inside a mechanical support structure composed of a yoke, four pads and a cylindrical outer shell. The description of the SMC structure and the reasons behind this mechanical design have been reported in [9].

\section{Model Constraints And PARAmeterization}

The superconducting cable features have been fixed at an early stage on the base of the NED specifications. The SMC Rutherford-type cable has 14 PIT strands with a diameter of 1.25 $\mathrm{mm}$. It has a rectangular section of $9.7 \times 2.2 \mathrm{~mm}$, plus $0.2 \mathrm{~mm}$ per face for the epoxy insulation in the baseline configuration, as shown in Fig. 1.

The target practical strand current density at $12 \mathrm{~T}$ and $4.2 \mathrm{~K}$ is $2500 \mathrm{~A} / \mathrm{mm}^{2}$, corresponding to an engineering current density $\mathrm{J}_{\text {eng }}$ around $654 \mathrm{~A} / \mathrm{mm}^{2}$. The critical current slope $\alpha_{c}$ has been 
TABLE I

SMC CABLE PROPERTIES

\begin{tabular}{lccc}
\hline \hline Parameter & Symbol & Unit & Value \\
\hline Number of strands & $N_{s t r}$ & $/$ & 14 \\
Strand diameter & $\emptyset_{s t r}$ & $\mathrm{~mm}$ & 1.25 \\
Cu/nCu rate & $r_{C u / n C u}$ & $/$ & 1.25 \\
Twist pitch & $p$ & $\mathrm{~mm}$ & 60 \\
Bare cable width & $w_{c b l}$ & $\mathrm{~mm}$ & 9.7 \\
Bare cable thickness at a stress of 20 MPa & $t_{c b l}$ & $\mathrm{~mm}$ & 2.20 \\
Epoxy insulation thickness per face & $t_{i n s}$ & $\mathrm{~mm}$ & 0.2 \\
Critical current slope at $12 \mathrm{~T}$ & $\alpha_{c}$ & $\mathrm{~A} / \mathrm{T}$ & -3435 \\
Critical current density slope at $12 \mathrm{~T}$ & $\alpha_{J}$ & $\mathrm{~A} / \mathrm{mm}^{2} / \mathrm{T}$ & -450 \\
Target current density at $12 \mathrm{~T}, 4.2 \mathrm{~K}$ & $J_{t r g}$ & $\mathrm{~A} / \mathrm{mm}^{2}$ & 2500 \\
\hline \hline
\end{tabular}

TABLE II

SMC MAGNETIC SPECIFICATIONS

\begin{tabular}{lccc}
\hline \hline Parameter & Symbol & Unit & Specified value \\
\hline Peak field localization & $/$ & $/$ & straight section \\
Peak field & $B_{\max }$ & $\mathrm{T}$ & 13 \\
Straight-end field difference & $\Delta B$ & $\mathrm{~T}$ & $\geq 0.5$ \\
Central field & $B_{0}$ & $\mathrm{~T}$ & none \\
Uniform field zone length at 1\% & $L_{u}{ }^{1 \%}$ & $\mathrm{~mm}$ & $\sim 60$ \\
Short-sample limit current & $I_{S S}$ & $\mathrm{kA}$ & $\leq 20$ \\
Overall length & $L_{t o t}$ & $\mathrm{~mm}$ & limited \\
Overall width & $w_{t o t}$ & $\mathrm{~mm}$ & limited \\
\hline \hline
\end{tabular}

linearized around $12 \mathrm{~T}$ [7]. Those properties are summarized in Table I. At a later stage, different insulation types such as an innovative ceramic insulation [10] could be used, implying slight changes in the magnetic design.

It is proposed to match the peak magnetic field region with the high stress zone, in the middle of the straight section, where the cable performance is not reduced by the winding deformation. Doing this, we make sure that the conductor is as much mechanically homogeneous as possible, and we try to control the mechanical effects that will affect the magnet training and performances. In the following study, the field magnitude $|B|$ is always considered.

The peak field $B_{\max }$, in the center of the straight section of the racetrack, should approach $13 \mathrm{~T}$ at short sample limit. The magnetic peak field on the ends $B_{\text {end }}$ should be at least $0.5 \mathrm{~T}$ lower than the one on the straight section center $B_{s s}$. The uniformity of the field in this zone should be better than $1 \%$ over a full twist pitch length $p$. There is no constraint on the central field $B_{0}$, neither on the field harmonics. In addition to these magnetic specifications, the working current is limited to $20 \mathrm{kA}$ by the available power supply. The coil dimensions should remain limited. Additionally, there are implicit cost constraints. Those specifications are summarized in Table II.

The following magnetic optimization is focused on: the overall number of turns $N_{t o t}$, the number of end spacers $N_{s}$ on each side, the spacers' axial lengths $L_{i}\left(i=\left[1 ; N_{s}\right]\right)$, the

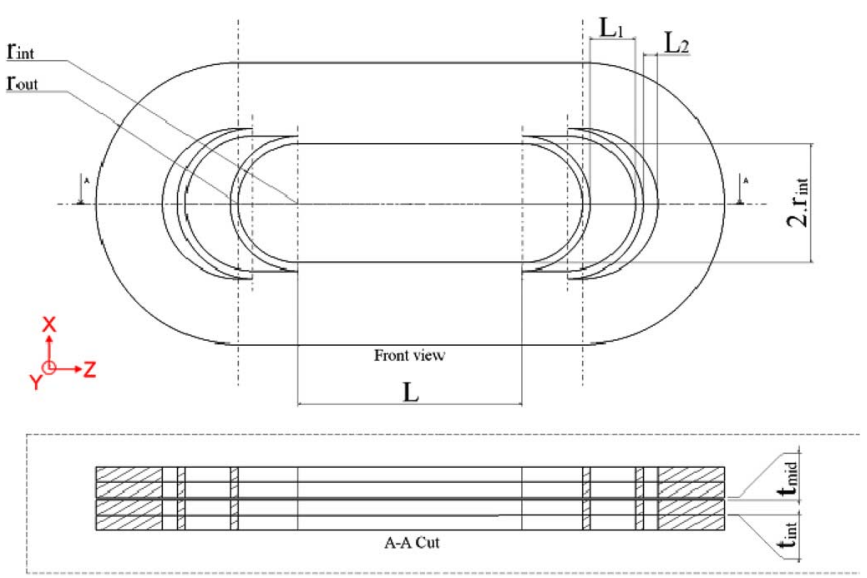

Fig. 2. SMC coil pack parameters.

number of turns in each block $N_{j}\left(j=\left[1 ; N_{s}+1\right]\right)$, the pole half-width $r_{i n t}$, the straight section length $L$, the interlayer insulation thickness $t_{i n t}$ and the mid-plane insulation thickness $t_{m i d}$. Those parameters are represented in Fig. 2 for a particular case.

\section{Modeling MethoD}

The magnetic optimization has been performed in parallel with the Finite Element Method codes CAST3M (CEA) [11], ANSYS (CERN), and OPERA (RAL) with frequent cross-checks. The duplicated calculations primarily served to validate the computations, and secondly they allowed the different institutes to benchmark the validity of their individual modeling processes.

The procedures used in CAST3M and OPERA are quite similar. The source field calculations are based on the semi-analytic Biot-Savart formulation, applied to the coil geometry divided into canonical sub-elements (bars and arcs) independent from the mesh. With iron, the Magnetic Scalar Potential (MSP) approach is considered, using the total scalar potential in regions which contain permeable materials (iron and air) and the reduced scalar potential in regions which contain source currents (coil and air). Both regions are fully meshed. The nonlinear iron characteristics are entered as a table of point values. On the interface surface between these regions, the two scalar potentials are linked together by applying the conditions of normal flux density and tangential magnetic field intensity continuity. The contributions of the current sources and the iron regions are added together in the post processing module to generate the final field distribution.

In ANSYS, two different approaches are followed: the Magnetic Vector Potential (MVP) and the MSP. The first approach is used for general assessment computations, whereas the second for the optimized configuration only. A model using the MVP formulation is faster to build and allows a direct computation of the Lorentz forces on the mesh. Nevertheless it could give incorrect results when nonlinear magnetic effects are included (iron saturation). The MSP approach allows performing faster simulations (one degree of freedom per element node instead of three), but it requires an electro-magnetic analysis in order to compute the coil current density to finally get the Lorentz forces. 


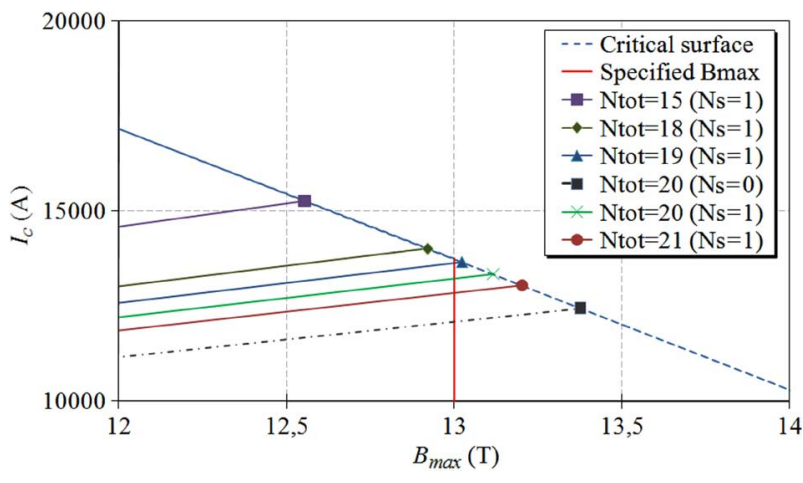

Fig. 3. Influence of $N_{t o t}$ on the magnet load lines with no spacer $\left(N_{s}=0\right)$ or with one spacer $\left(N_{s}=1, L_{1}=10 \mathrm{~mm}, N_{1}=2\right)$. No iron is considered here. The other parameters are set as listed in [12].

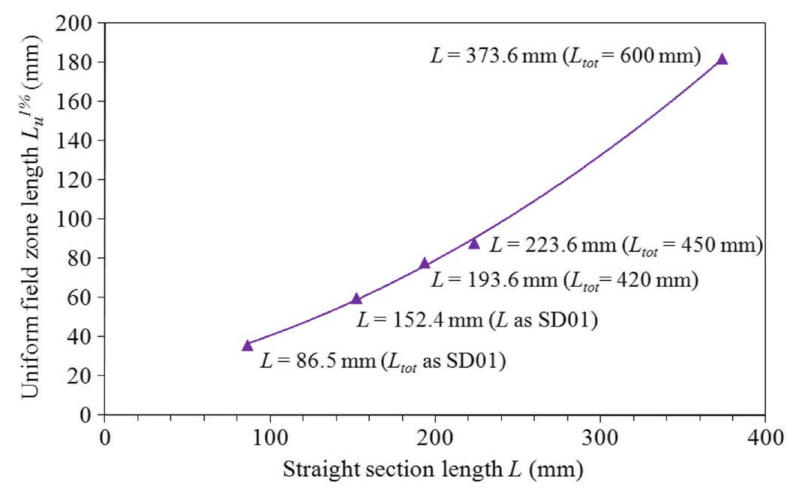

Fig. 4. Influence of $L$ on the uniform field zone length $L_{u}^{1 \%}$.

\section{PARAMETRIC STUDY}

\section{A. Number of Turns}

The influence of $N_{t o t}$ on $B_{\max }$ is studied on a baseline set of parameters inspired from SD01. Without end spacer, $N_{t o t} \geq 17$ is necessary for $B_{\max }$ to reach $13 \mathrm{~T}$. In this case, the peak field is located in the ends. With one spacer, $N_{\text {tot }}$ must be larger than 19, as showed on Fig. 3. For instance, with $N_{t o t}=20$, the use of one end spacer reduces $B_{\max }$ by about $0.25 \mathrm{~T}$.

\section{B. Coil Pack Parameters}

It is showed that $r_{i n t}$ should be as small as possible in terms of magnetic efficiency. For a fixed $N_{t o t}$, a smaller $r_{i n t}$ leads to higher $B_{\max }$ with lower $\Delta B$ [12]. On the other hand, $r_{\text {int }}$ needs to be large enough to avoid bending issues, such as cable popouts that could damage the cable. From the experience of SD01, we consider that $32.8 \mathrm{~mm}$ is the minimal value (by scaling $r_{i n t}$ with respect to the cable thickness ratio). We decide to allow a safety margin by setting $r_{\text {int }}$ to $40 \mathrm{~mm}$.

Fig. 4 shows that increasing $L$ reduces the effect of the ends on the straight section and improves the field uniformity. On the other hand, $L$ must remain short enough to make sure that the coil pack fits inside the furnace available. $L$ is fixed to $150 \mathrm{~mm}$ so that $L_{u}^{1 \%} \sim p$.

The insulation thicknesses are shown to have a second order influence on the resulting field. For practical reasons, $t_{m i d}$ is fixed to $1.6 \mathrm{~mm}$ and $t_{\text {int }}$ to $0.2 \mathrm{~mm}$. At this stage, the peak field remains located in the ends.
TABLE III

END SPACERS INFLUENCE ON MAGNETIC RESULTS

\begin{tabular}{ccccc}
\hline \hline Parameter & Unit & $N_{s}=0$ & $N_{s}=1$ & $N_{s}=2$ \\
\hline$L_{l}$ & $\mathrm{~mm}$ & $/$ & 40 & 30 \\
$L_{2}$ & $\mathrm{~mm}$ & $/$ & $/$ & 10 \\
\hline$B_{s s}$ & $\mathrm{~T}$ & 11.41 & 11.10 & 11.11 \\
$B_{\text {end }}$ & $\mathrm{T}$ & 13.78 & 12.51 & 11.66 \\
$\Delta B$ & $\mathrm{~T}$ & -2.37 & -1.41 & -0.55 \\
\hline \hline
\end{tabular}

TABLE IV

IRON PARTS INFLUENCE ON MAGNETIC RESULTS

\begin{tabular}{cccccc}
\hline \hline Parameter & Unit & Set 0 & Set 1 & Set 2 & Set 3 \\
\hline$B_{s S}$ & $\mathrm{~T}$ & 12.00 & 12.16 & 12.91 & 12.94 \\
$B_{\text {end }}$ & $\mathrm{T}$ & 12.60 & 12.69 & 12.26 & 12.27 \\
$\Delta B$ & $\mathrm{~T}$ & -0.60 & -0.53 & 0.64 & 0.67 \\
\hline \hline
\end{tabular}

TABLE V

SMC OPTIMIZED PARAMETERS

\begin{tabular}{lccc}
\hline \hline Parameter & Symbol & Unit & Value \\
\hline Number of turns & $N_{\text {tot }}$ & $/$ & 21 \\
Number of end spacers & $N_{s}$ & $/$ & 2 \\
Axial length of the inner spacer & $L_{l}$ & $\mathrm{~mm}$ & 30 \\
Axial length of the outer spacer & $L_{2}$ & $\mathrm{~mm}$ & 10 \\
Number of turns in the inner block & $N_{I}$ & $/$ & 2 \\
Number of turns in the mid-block & $N_{2}$ & $/$ & 2 \\
Pole half-width & $r_{i n t}$ & $\mathrm{~mm}$ & 40 \\
Straight section length & $L$ & $\mathrm{~mm}$ & 150 \\
Interlayer insulation thickness & $t_{\text {int }}$ & $\mathrm{mm}$ & 0.2 \\
Mid-plane insulation thickness & $t_{\text {mid }}$ & $\mathrm{mm}$ & 1.6 \\
\hline \hline
\end{tabular}

\section{End Spacers}

It is proposed to add spacers in the magnet ends. Doing this, we expect to decrease significantly $B_{\text {end }}$ while keeping $B_{s s}$ almost unchanged. Configurations with zero, one, or two end spacers per side are considered. For a fixed current of $14 \mathrm{kA}$, Table III shows that the most efficient configuration in terms of $\Delta B$ is for $N_{s}=2 . N_{t o t}$ is fixed to 21 . The best configuration is obtained for $N_{1}=N_{2}=2$ (i.e. $N_{3}=17$ ), with $L_{1}=30 \mathrm{~mm}$ and $L_{2}=10 \mathrm{~mm}$ [12]. The peak field is still located in the ends.

\section{Iron Parts}

Using iron parts along the straight section of the magnet will help concentrate the flux lines in this region. It is proposed to take advantage of the SMC structure by making some of its parts in MAGNETIL iron. Different cases are investigated: no iron (set 0 ); iron yoke (set 1 ); iron yoke and vertical pads (set 2 ); iron yoke and all pads (set 3 ). Contrary to SD01, a non-magnetic pole is preferred. In every case, the iron covers $70 \%$ of $L$, i.e. 105 $\mathrm{mm}$. Table IV shows at $I_{s s}$ that the simplest suitable solution is obtained with the set 2 .

The previous parametric study leads to the final set of parameters presented in Table V. 
TABLE VI

SMC MAGNETIC RESULTS COMPARISON

\begin{tabular}{ccccccc}
\hline \hline \multirow{2}{*}{ Parameter } & \multirow{2}{*}{ Unit } & \multirow{2}{*}{ Target } & $\begin{array}{c}\text { CAST3M } \\
\text { MSP }\end{array}$ & $\begin{array}{c}\text { OPERA } \\
\text { MSP }\end{array}$ & $\begin{array}{c}\text { ANSYS } \\
\text { MSP }\end{array}$ & $\begin{array}{c}\text { ANSYS } \\
\text { MVP }\end{array}$ \\
\hline$B_{\text {max }}$ & $\mathrm{T}$ & 13 & 12.92 & 12.96 & 12.94 & 12.85 \\
$B_{\text {end }}$ & $\mathrm{T}$ & $\leq 12.5$ & 12.22 & 12.23 & 12.22 & 12.20 \\
$\Delta B$ & $\mathrm{~T}$ & $\geq 0.5$ & 0.70 & 0.73 & 0.71 & 0.71 \\
$B_{0}$ & $\mathrm{~T}$ & none & 9.93 & 9.80 & 9.65 & 9.85 \\
$L_{u}{ }^{1 \%}$ & $\mathrm{~mm}$ & $\sim 60$ & $\sim 70$ & $\sim 60$ & $\sim 55$ & $\sim 55$ \\
\hline$I_{s S}$ & $\mathrm{kA}$ & $\leq 20$ & 14.01 & 14.00 & 13.96 & 14.25 \\
\hline Forces $\left(F_{X}\right)$ & $\mathrm{MN} / \mathrm{m}$ & & 1.9 & 2.0 & 2.0 & 2.1 \\
Forces $\left(F_{Y}\right)$ & $\mathrm{MN} / \mathrm{m}$ & & -1.8 & -1.9 & -1.9 & -1.9 \\
Stored energy & $\mathrm{kJ}$ & & 211 & 209 & 190 & 198 \\
Inductance & $\mathrm{mH}$ & & 2.1 & 2.1 & 1.9 & 2.0 \\
\hline \hline
\end{tabular}

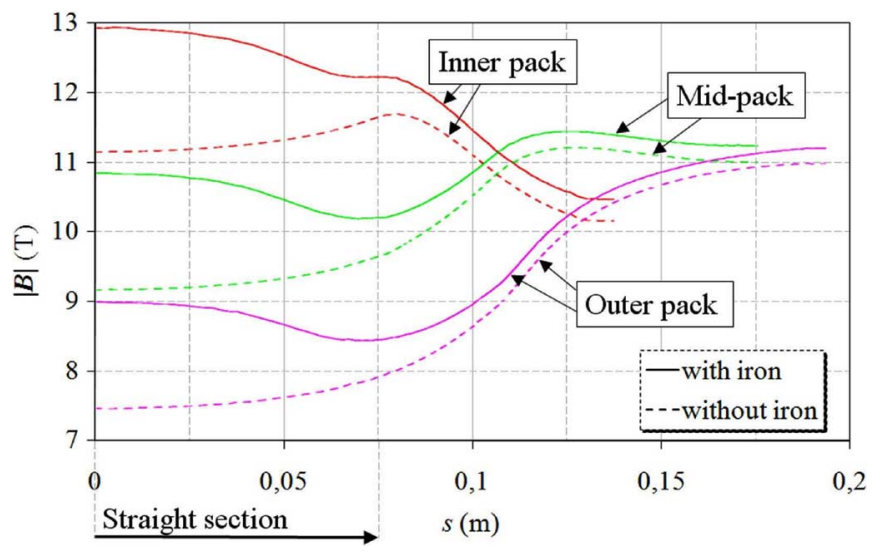

Fig. 5. Magnetic field magnitude distribution inside the coil along turn 1 (inner pack), turn 3 (mid-pack) and turn 5 (outer pack) at $I_{s s}$ with and without iron parts (CAST3M). $s$ is the curvilinear abscissa along the cable; $s=0$ in the center of the straight section of the magnet.

\section{FinAl Results AND Code Benchmarking}

The magnet design process was an iterative one, with the different laboratories using four different magnetic design formulations. The previous analysis has voluntarily been presented straight-forward, without pointing the codes used. The final magnetic results, based on the optimized set of parameters, are compared in Table VI.

A first glance shows a good agreement between all four models. Discrepancies below $1 \%$ are observed on $B_{\max }$ and $B_{\text {end }}$, and below $3 \%$ on $B_{0}$. They can be explained partly by the mesh effect influencing the local peak values, and partly by the formulation choice [12]. The discrepancy is lower between the three MSP models, around $0.3 \%$. This can be explained by the source conductor definition which follows the same semi-analytical approach in those cases [13]. Despite the differences in magnetic formulations, model features, and numerical solvers, the results appear to be in very good agreement everywhere on the coil. The four models lead to the same optimized solution. The field magnitude distribution inside the coil is represented in Fig. 5 along three particular turns. This plot highlights the influence of the iron parts on the peak field location.

\section{CONCLUSION}

The magnetic optimization of the SMC racetrack magnet has been presented on the base of the NED cable. Using the SD01 subscale dipole as a starting point, the SMC study has shown: optimizing the number of turns and the coil pack features; using two end spacers on each side; using a non-magnetic pole; optimizing the iron parts. The resulting magnetic field has a maximum around $13 \mathrm{~T}$ located as desired on the dipole straight section, for a quench current around $14 \mathrm{kA}$. This peak field shows a significant margin compared to the ends (over $0.7 \mathrm{~T}$ ) and a good uniformity over a twist pitch. The final coil pack is approximately $420 \mathrm{~mm}$-long, $190 \mathrm{~mm}$-wide and $42 \mathrm{~mm}$-thick. This configuration is ideal for the future training studies with variable mechanical pre-stresses.

The magnetic field computations have been cross-checked between CAST3M, OPERA, and ANSYS (MSP and MVP formulations). Those four nonlinear models lead to the same conclusions in terms of magnet design.

The next step has been to calculate and to design an adapted mechanical test bed. This structure has been built and instrumented. It is being calibrated, before the first full magnet tests programmed for the beginning of 2010 .

\section{ACKNOWLEDGMENT}

The authors would like to thank Arnaud Devred (ITER), Hélène Félice, Paolo Ferracin, Shlomo Caspi, Ray Hafalia (LBNL) and François-Paul Juster (CEA/IRFU).

\section{REFERENCES}

[1] A. Lietzke et al., "Test results of $\mathrm{RD} 3 \mathrm{c}, \mathrm{a} \mathrm{Nb}_{3} \mathrm{Sn}$ common-coil racetrack dipole magnet," IEEE Trans. Applied Superconductivity, vol. 13, pt. 2, pp. 1292-1296, 2003.

[2] S. Mattafirri et al., "Performance analysis of HD1: a $16 \mathrm{~T} \mathrm{Nb}_{3} \mathrm{Sn}$ dipole magnet," IEEE Trans. Applied Superconductivity, vol. 15, pt. 2, pp. 1156-1159, 2005.

[3] S. Caspi et al., "Test results of LARP $\mathrm{Nb}_{3} \mathrm{Sn}$ quadrupole magnets using a shell-based support structure (TQS)," IEEE Trans. Applied Superconductivity, vol. 19, no. 3, pt. 2, pp. 1221-1225, June 2009.

[4] A. Zlobin et al., "R\&D of $\mathrm{Nb}_{3} \mathrm{Sn}$ accelerator magnets at Fermilab," IEEE Trans. Applied Superconductivity, vol. 15, pt. 2, pp. 1113-1118, 2005.

[5] A. Devred et al., "Overview and status of the Next European Dipole (NED) joint research activity," Supercond. Sci. Technol., vol. 19, 2006.

[6] T. Boutboul et al., "Nb3Sn conductor development and characterization for NED," J. Phys.: Conf. Ser., vol. 97, 2008.

[7] A. Devred et al., "Status of NED conductor development," presented at the IEEE/CSC \& ESAS Europ. Superconductivity News Forum, 2007, ST5.

[8] H. Félice, "Design and test of a $\mathrm{Nb}_{3} \mathrm{Sn}$ subscale dipole magnet for training studies," IEEE Trans. Applied Superconductivity, vol. 17, pt. 2, pp. 1144-1148, 2007.

[9] F. Regis et al., "Mechanical design of the SMC (Short Model Coil) dipole magnet," IEEE Trans. Applied Superconductivity, to be published.

[10] F. Rondeaux, "Insulation development: Final report on innovative insulation," CARE Report, 2007-037-NED, 2007.

[11] P. Verpeaux, T. Charras, and A. Millard, "Castem2000: une approche moderne du calcul des structures," in Calcul des Structures et Intelligence Artificielle. : : Pluralis, 1988, p. 261.

[12] P. Manil, F. Regis, and J. Rochford et al., "NED short model coil project: technical note on magnetic design," CEA/CERN, EDMS id: 926137, 2008, Technical Report.

[13] M. Gyimesi, D. Lavers, T. Pawlak, and D. Ostergaard, "Biot-Savart integration for bars and arcs," IEEE Trans. Magnetics, vol. 29, no. 6, pp. 2389-2391, 1993. 